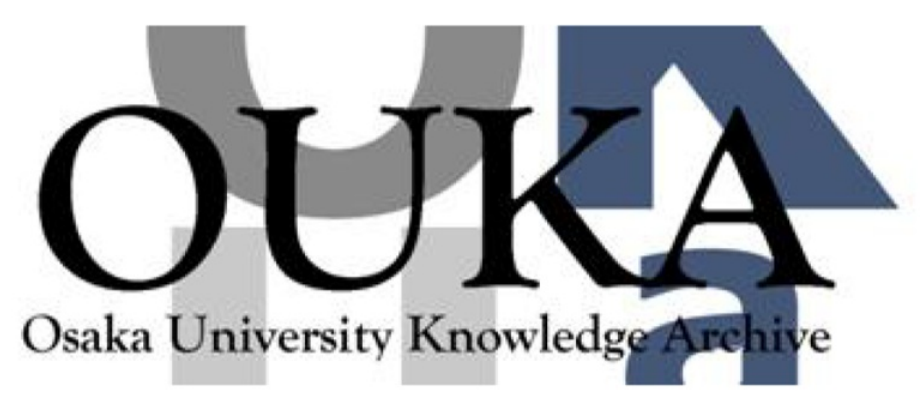

\begin{tabular}{|c|c|}
\hline Title & $\begin{array}{l}\text { Thin, high atomic weight refractory film } \\
\text { deposition for diffusion barrier, adhesion } \\
\text { layer, and seed layer applications }\end{array}$ \\
\hline Author (s) & $\begin{array}{l}\text { Rossnagel, S. M.; Nichols, C.; Hamaguchi, S. et } \\
\text { al. }\end{array}$ \\
\hline Citation & $\begin{array}{l}\text { Journal of Vacuum Science and Technology B: } \\
\text { Microelectronics and Nanometer Structures. } \\
\text { 14(3) p. 1819-p. } 1827\end{array}$ \\
\hline Issue Date & $1996-03$ \\
\hline oaire:version & VoR \\
\hline URL & https://hdl. handle. net/11094/78502 \\
\hline rights & $\begin{array}{l}\text { This article may be downloaded for personal use } \\
\text { only. Any other use requires prior permission } \\
\text { of the author and AIP Publishing. This article } \\
\text { appeared in Journal of Vacuum Science \& } \\
\text { Technology B: Microelectronics and Nanometer } \\
\text { Structures Processing, Measurement, and } \\
\text { Phenomena 14, 1819 (1996) and may be found at } \\
\text { https://doi.org/10.1116/1.588562. }\end{array}$ \\
\hline Note & \\
\hline
\end{tabular}

Osaka University Knowledge Archive : OUKA

https://ir. Library. osaka-u. ac. jp/

Osaka University 


\title{
Thin, high atomic weight refractory film deposition for diffusion barrier, adhesion layer, and seed layer applications
}

\author{
S. M. Rossnagel, C. Nichols, ${ }^{\text {a) }}$ S. Hamaguchi, D. Ruzic, ${ }^{\text {b) }}$ and R. Turkot ${ }^{\text {b) }}$ \\ T. J. Watson Research Center, IBM, P.O. 218, Yorktown Heights, New York 10598
}

(Received 2 January 1996; accepted 2 April 1996)

\begin{abstract}
Thin, nearly conformal films are required for semiconductor applications to function as diffusion barriers, adhesion layers and seed layers within trenches and vias. The deposition of high mass refractory films with conventional, noncollimated magnetron sputtering at low pressures shows better-than-expected conformality which is dependent on the degree of directionality of the depositing atoms: the conformality increases as the directionality increases. The primary cause appears to be a strongly angle-dependent reflection coefficient for the depositing metal atoms. As the deposition is made more.directional by increasing the cathode-to-sample distance, the depositing atoms are more likely to reflect from the steep sidewalls, leading to better conformality as well as a less columnar film structure. (C) 1996 American Vacuum Society.
\end{abstract}

\section{INTRODUCTION}

Diffusion barrier thin films are used routinely in semiconductor applications to separate potentially reactive materials. The potential chemical reactions may occur spontaneously under deposition conditions or may occur later during either subsequent film processing, perhaps at higher temperature, or during operation in the lifetime of the circuit, leading to reliability problems. A common diffusion barrier used in interconnect applications is $\mathrm{TiN}$, which is used to protect $\mathrm{SiO}_{2}$ trench walls from chemical attack by $\mathrm{WF}_{6}$ during chemical vapor deposition $\mathrm{W}$ deposition, and also to chemically separate the deposited $\mathrm{W}$ from reaction with either $\mathrm{Al}$, $\mathrm{Si}$, or silicides.

Diffusion barriers must be chemically inert themselves as well as moderately conductive. TiN, for example, is very inert. stable, and has an as-deposited resistivity in the range of 4 () to $150 \mu \Omega \mathrm{cm}$. Diffusion barrier thin films must also be conformal, thin, and have low porosity. This latter requirement leads to a desire for an amorphous material. or at least one which is not characterized by a very columnar structure. characteristic of a zone 1 film in the Thornton zone diagram. ${ }^{\prime}$

The materials set used for diffusion barriers varies by application. In addition. often diffusion barriers are used either as adhesion layers or in combination with adhesion layers. The materials often used include Ti, TiN, TiW, Ta. TaN, Cr. $\mathrm{Si}_{3} \mathrm{~N}_{4}$. and good reviews of this general area and applications are available. ${ }^{2-5}$ The particular materials used, though, will strongly depend on the specific material system used. the deposition conditions and subsequent processing. Physical sputtering, typically with magnetron cathodes, is commonly used for the deposition of films such as Ti. TiN, TiW, $\mathrm{Ta}, \mathrm{TaN}, \mathrm{Cr}$, etc. which are easily fabricated into sputtering targets and have a reasonable sputter yield and deposition rate.

As the aspect ratio (AR, defined as the feature depth di-

\footnotetext{
"Permanent address: Dept. of Applied Sciences, College of William and Mary, Williamsburg, VA.

b'Also at Dept. of Nuclear Engineering. University of Illinois. Urbana, IL.
}

vided by the feature width) of semiconductor features has increased, conventional sputtering has become less useful for depositing diffusion barriers, adhesion layers and seed layers because of the large, nonnormal incidence component to the depositing flux. These high-angle depositing atoms tend to form overhangs at the top corners of high aspect ratio vias and trenches and this constriction causes later problems with the deposition or filling process for the trench or via. A solution to this problem was proposed which used a physical collimator or filter interposed between the magnetron cathode and the sample. ${ }^{6.7}$ The collimator tends to collect the sputtered atoms which are not moving at near normal incidence allowing the mostly normal incidence atoms to pass through the collimator and deposit on the sample. This increase in directionality is useful in reducing the overhang formation and allowing some deposition into the trench or via.

Diffusion barriers deposited by collimated sputtering have a characteristic profile, as shown in Fig. 1. The depositing flux, which is now arriving at the feature surface with a limited angular distribution, has a much higher "step coverage" on the bottom surface of the feature. Step coverage in this case is defined as the local film thickness divided by the thickness of the deposited film on the broad, flat areas near the trench or via feature, and can range from 0 to $100 \%$. The step coverage on the bottom of the feature is dependent on the aspect ratio of the collimator, and can approach nearly $100 \%$ at very high collimator aspect ratios. At the same time, however, the sidewall step coverage decreases as the collimator aspect ratio increases, approaching zero at very high collimator aspect ratios. Collimator aspect ratio is defined as the physical thickness of the collimator divided by the opening diameter of each collimator hole.

The deposition on the walls of the features using moderate collimation has a slowly undercutting profile, as seen in Fig. 1. Since the depositing flux is highly directional, locations further down the sidewall have a reduced deposition rate due to the self shadowing of the upper wall. This can 
Collimator

\section{IIIIIIIIIIIIII}

Depositing flux $\mid \begin{array}{lllll}1 & 1 & 1 & 1 & 1\end{array} 1$

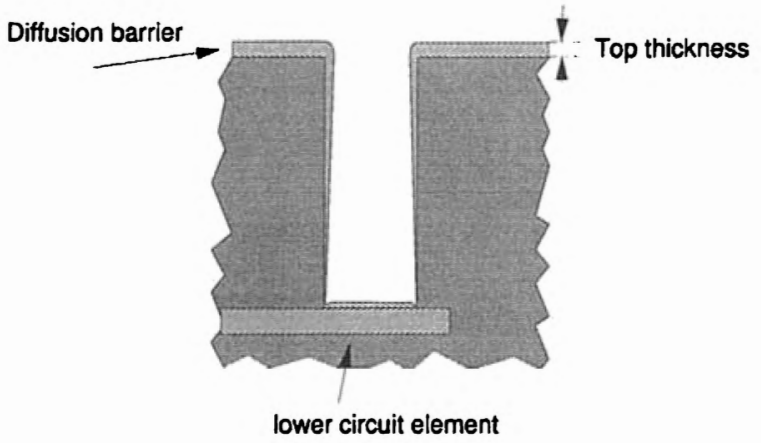

FiG. 1. Sketch of the deposition profile of a thin film diffusion barrier deposited into a trench by collimated sputter deposition.

reach an extreme in the bottom corner of the deposit where the step coverage can be only a few percent. This lower corner is also often characterized by a crack between the denser bottom deposit and the less dense film on the sidewall, which is clearly undesirable. In addition, in the case of $\mathrm{Ti}$ and $\mathrm{TiN}$, the films deposited on the sidewalls are quite columnar and nodular due to the high reactivity and sticking of the depositing atoms. To help repair some of these problems, samples are often overdeposited to provide adequate coverage on the sidewalls. In addition samples can be annealed at moderate temperatures $\left(400^{\circ} \mathrm{C}\right)$ or multiple layers can be deposited under slightly different process conditions to help overcome the possibility of voids or cracks permeating the film. ${ }^{8}$

Collimated sputtering, however, is fairly slow and expensive due to the poor efficiency of the collimator and related problems such as collimator lifetime, flaking, uniformity changes, etc. Other techniques, such as ionized physical vapor deposition (I-PVD) may be useful in eliminating the collimator and yet providing a controlled directional deposition through the condensation of ions directly from a metal plasma to form a film. ${ }^{9-13}$ It has been observed, though, that for some refractory metal systems (e.g., Ta, W), the deposited film profile of uncollimated, nonionized material is somewhat better than expected, in that the film shows less overhang formation during conventional sputter deposition than is observed for lower mass species (Ti, Al, Cu, etc.). ${ }^{14}$ This report examines conventional, noncollimated sputter deposition of high mass refractory materials to determine what phenomena may be contributing to the deposition profiles observed.

\section{EXPERIMENT}

Samples were prepared of typical interconnectlike features. The samples were $\mathrm{Si}$ wafers with a thermal oxide

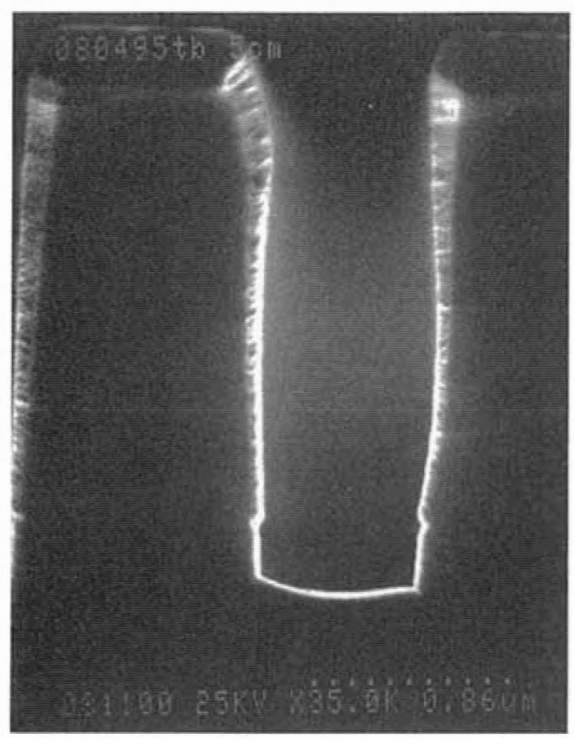

FIG. 2. SEM micrograph of a $1500 \AA$ Ta film deposited by conventional sputtering at a cathode-to-sample distance (throw) of $5 \mathrm{~cm}$. The deposition pressure was $1 \mathrm{mTorr}$ in $\mathrm{Ar}$ and the magnetron power was $2.5 \mathrm{~kW}$.

thickness of approximately $2 \mu \mathrm{m}$. Arrays of trenches, some of which were very long and others only $2-3$ trenchwidths in length as well as square vias were fabricated in the surface of the oxide to a depth of $1.9 \mu \mathrm{m}$. The feature width varied from 0.5 to over $5 \mu \mathrm{m}$, with a maximum aspect ratio of nearly 4 . The films were deposited into these features using conventional magnetron sputtering, with commercial cathodes (Applied Materials Endura class; circular planar cathode with a diameter of $30 \mathrm{~cm}$ ) with rotating magnetdefined erosion paths for better uniformity and cathode utilization. The $200 \mathrm{~mm}$ wafer samples were deposited at room temperature in Ar at pressures of $1 \mathrm{mTorr}$ or less. The cathode-tosample or "throw" distance could be varied from 5 to $35 \mathrm{~cm}$. No sample cleaning or preparation was done. Sample analysis was primarily by high resolution scanning electron microscopy (SEM). Typically, film thicknesses of 1000 to 2000 $\AA$ were deposited on the top surface. The step coverage. or relative deposition, at various locations in the trench and via features were measured by examination of the SEM photographs.

An example of a short $(5 \mathrm{~cm})$ throw distance, high aspect ratio feature is shown in Fig. 2. Consistent with the fairly broad angular distribution in the sputtered flux, there is considerable thickening and overhang formation visible at the top edges of the trench. As the cathode to sample distance is increased, the profiles of the deposited films gradually change. At a distance of $15 \mathrm{~cm}$, the deposition is more conformal on the sidewalls (Fig. 3). At the longest distance routinely used, $25 \mathrm{~cm}$, the film thickness on the sidewalls was quite uniform down the sidewall and little evidence of overhang formation was seen (Fig. 4).

The measured step coverage as a function of sidewall position is shown in Fig. 5 as a function of the cathode-10sample distance for 2.8:1 aspect ratio, 0.5 - $\mu \mathrm{m}$-wide trench features. The bottom thickness as a function of throw dis- 


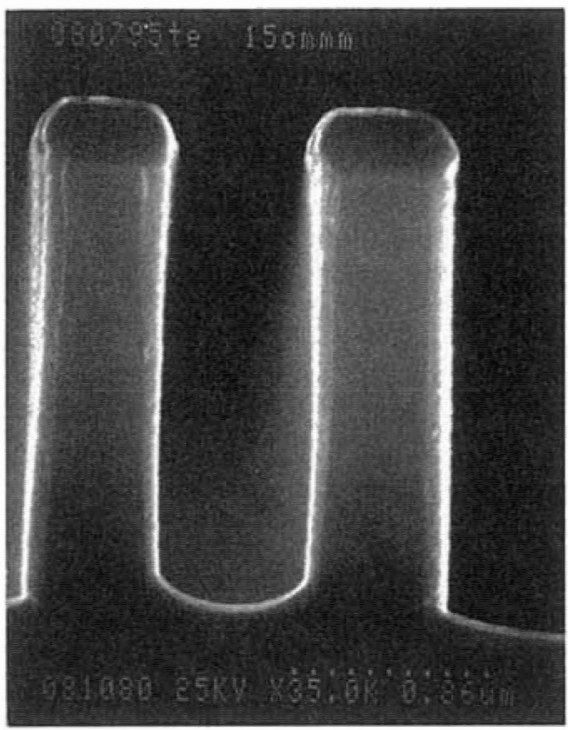

FIG. 3. SEM micrograph of a $1500 \AA \AA$ Ta deposited under identical conditions (1) Fig. 2 with a cathode-to-sample distance of $15 \mathrm{~cm}$.

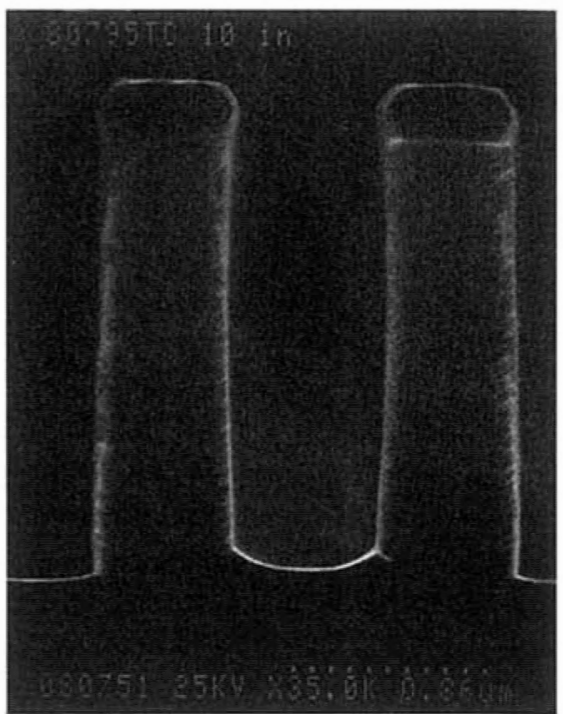

FiG. 4. SEM micrograph of a $1800 \AA$ Ta film deposited under conditions identical to Figs. 2 and 3 with a cathode to sample distance of $25 \mathrm{~cm}$.

\section{Step Coverage Down Sidewall}

Slep Coverage

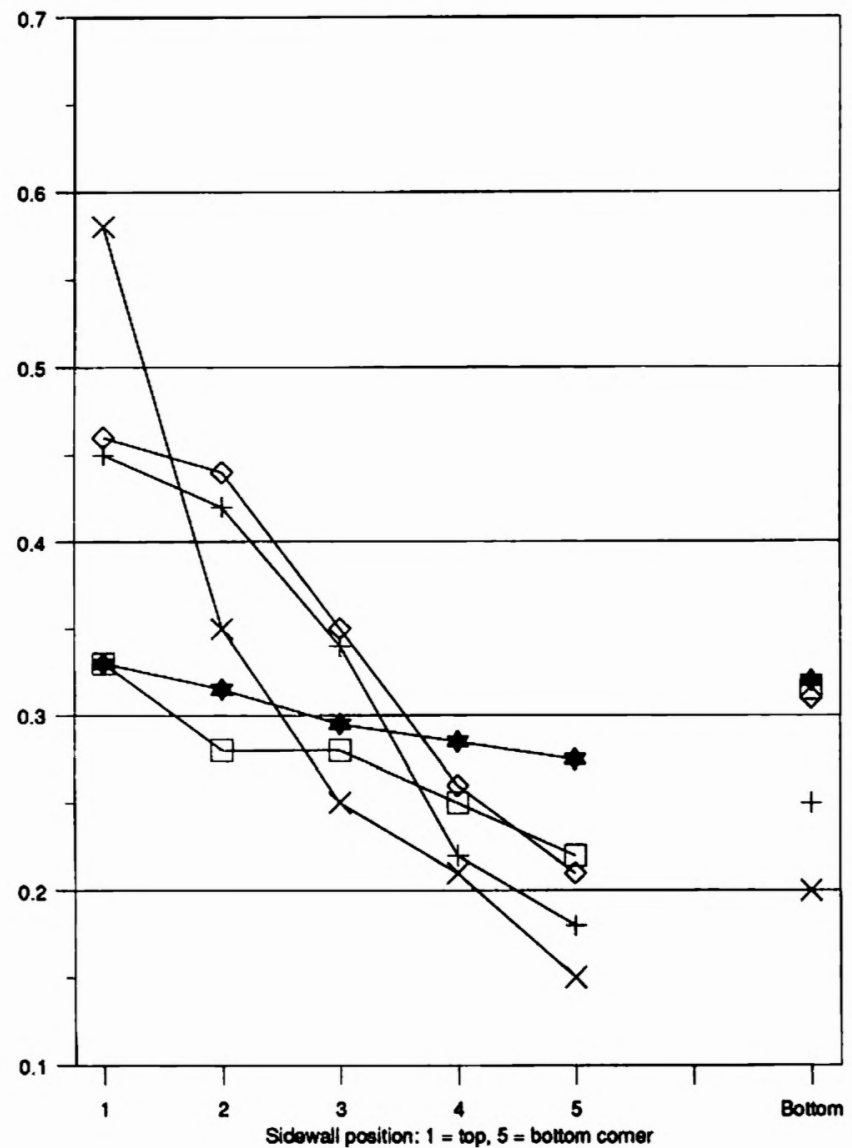

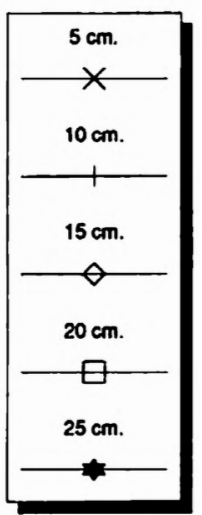

tiom

FiG. 5 . The step coverage of Ta films deposited on the sidewalls of 2.8:1 AR. $0.5 \mu \mathrm{m}$ trenches as a function of sidewall position for throw distances of 5,10 , 15. 20. and $25 \mathrm{~cm}$. The bottom coverage in each case is also shown. Step coverage is defined as the local film thickness divided by the thickness of the film deposited on the wide. flat top areas near the trench feature. 


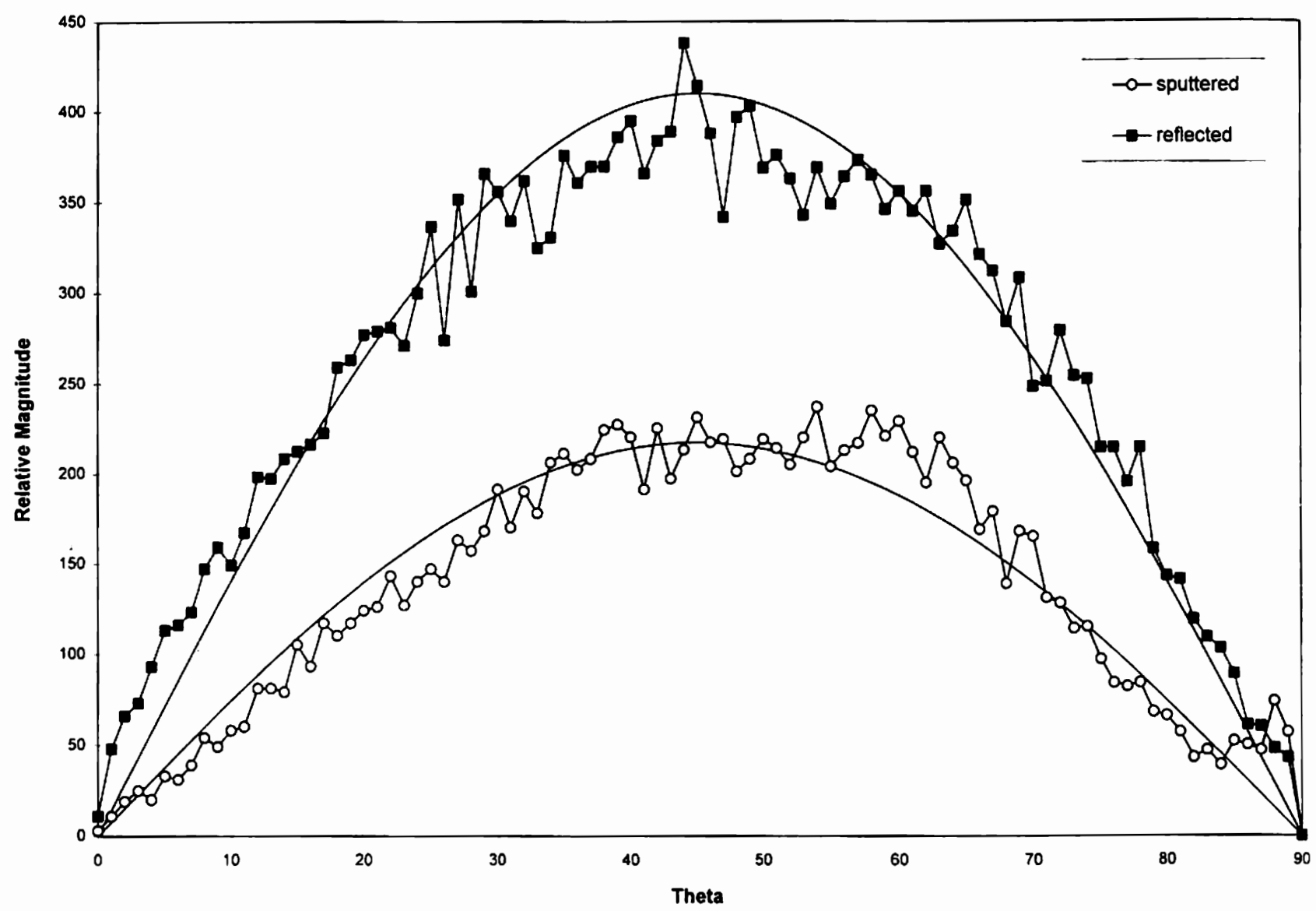

Fig. 6. Angular emission distribution calculated using fractal TRIM for $400 \mathrm{eV}$ Ar sputtering of Ta (open circles). Also shown is the emission distribution for reflected Ar neutrals (solid squares). The relative magnitude ( $y$-axis) is in arbitrary units and is included to show that the number of reflected Ar atoms is roughly $2 \times$ the number of sputtered Ta atoms. The solid lines in each case are cosine-theta traces, which in polar geometries are actually sine-theta cosine-theta distributions.

tance is also shown. Several trends can be observed in this figure. At short throw distances, the profile is strongly tapered and the step coverage at the bottom corner is very low. As the throw distance is increased, the sidewall thickness becomes more uniform. In addition, there is an increase in the lower sidewall film thickness, inconsistent with a completely directional deposition. It should be noted that increasing the cathode to sample throw distance is functionally equivalent to interposing a collimator between the cathode and sample, at least for samples located on the centerline of the system. While collimation was not used in this experiment, a throw distance of $25 \mathrm{~cm}$ is approximately equal to an interposed collimator of aspect ratio near 1.0.

\section{DISCUSSION}

The deposition profiles observed in Figs. 2-5 are generally inconsistent with conventional, noncollimated sputtering in that they show (1) better than expected conformality or lowerwall step coverage, and (2) an increase in lower wall step coverage with increasing directionality (i.e., throw distance). With conventional sputtering, a very steeply undercut wall step coverage should be observed with virtually zero coverage at distances greater than one trench-width from the top of the trench. Increased directionality should reduce the overhang formation, but should also result in proportionately higher bottom step coverage (with increasing distance or directionality) as well as a significantly reduced sidewall coverage. At the longer throw distances, it should be noted that the bottom and sidewall step coverages are fairly similar. This is also inconsistent with a high-directionality deposition which would lead to very high levels of bottom surface step coverage but virtually zero lower wall coverage.

Two physical effects could be contributing to these experimental observations. First, if the emission profile of the sputtered atoms was very highly forward-peaked, the deposition might have similar characteristics to a collimated deposition. This same effect has been observed in single crystal cathodes which show a preferred, nearnormal incidence emission pattern. ${ }^{15}$ Second, if the depositing atoms showed any degree of reflection from the sidewalls of the deposition, the reflected and redeposited flux would tend to be more conformal due to the local redeposition. The samples were at near room temperature for these depositions and surface diffusion of the refractory materials is not expected to be significant.

Physical sputter deposition of a high mass species, such as 


\section{$\mathrm{Rn} \quad \mathrm{Ta}$ Reflection from SiO2 and $\mathrm{Ta}$}

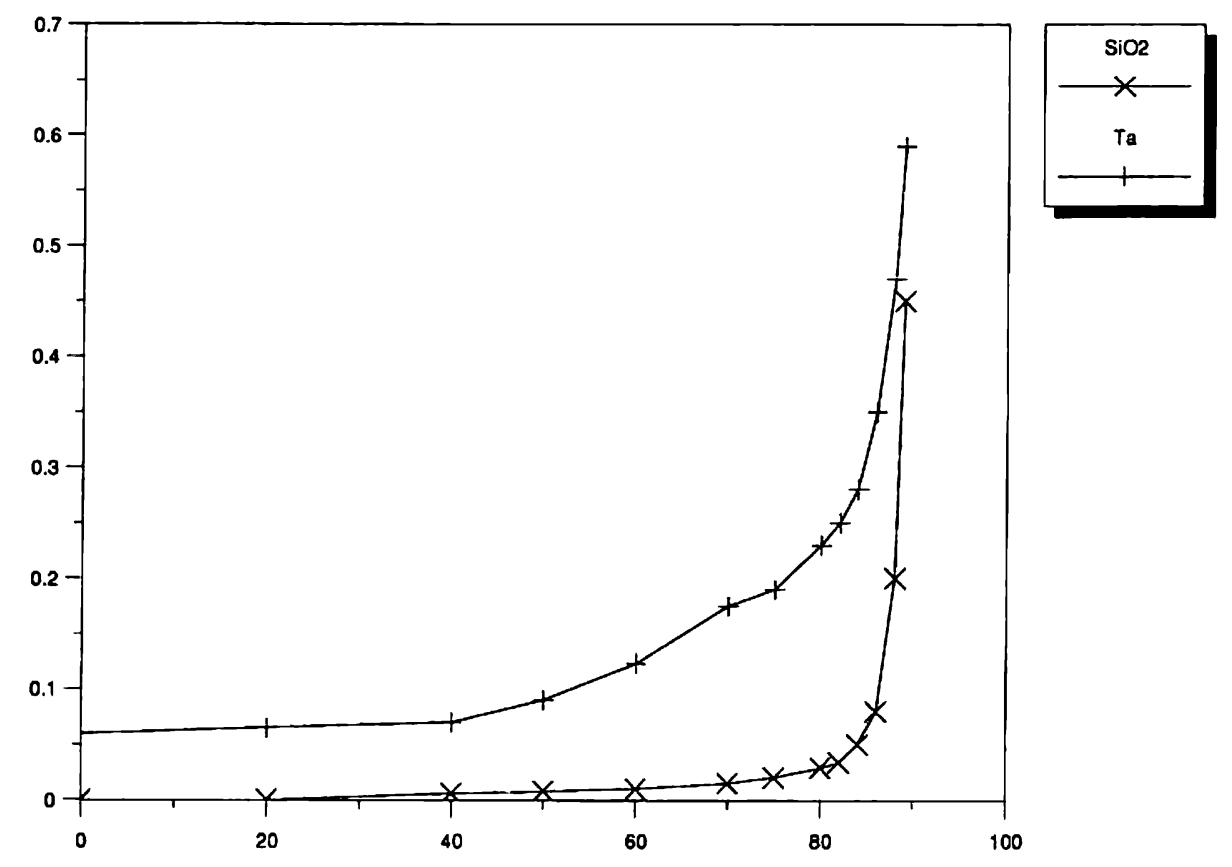

Angle of Incidence

FIG. 7. The reflection coefficient, $R n$, for $25 \mathrm{eV}$ Ta incident on a $\mathrm{Ta}(+)$ and quartz $(X)$ surface as a function of incident angle. In this configuration, $0^{\circ}$ is normal incidence.

$\mathrm{Ta}$ and $\mathrm{W}$, has been routinely used and such issues as the energetics and sputter yields have been known for decades. High mass refractory materials have moderate yields in $\mathrm{Ar}$ at magnetron voltages $(300-600 \mathrm{~V})$ of 0.3 to 0.5 atoms/ion. The emitted atoms, however, have long been thought to have quitc high kinetic energy, based on this early work. ${ }^{16}$ Kinetic energies of nearly $100 \mathrm{eV}$ per sputtered atom were originally reported. It is likely that this work underestimated the effect of energetic, reflected neutrals (Ar) on the energy deposition at the sample surface, as kinetically it seems difficult to explain the transfer of energy from the incident $400 \mathrm{eV}$ Ar to a single $100 \mathrm{eV}$ Ta or $\mathrm{W}$ sputtered atom.

\section{A. TRIM modeling}

The dynamics of Ar sputtering of Ta were explored using a variant of the TRIM program which has been modified for fractal-like surfaces. ${ }^{17.18}$ This modification allows more accurate predictions of the angular emission profiles, particularly at low angles, where a nonplanar surface may lead to recapture of some of the emitted atoms. The angular distribution of Ta sputtered with $400 \mathrm{eV} \mathrm{Ar}$ is shown in Fig. 6. The emission profile is close to a cosine distribution (solid line). which suggests no preferential or peaked emission. The averalge kinetic energy of the $\mathrm{Ta}$ is about $26 \mathrm{eV}$, about a factor of $4 \times$ reduced from the original Wehner work, ${ }^{16}$ and perhaps more consistent with lower mass species, such as $\mathrm{Cu}$, which have average energies of $10 \mathrm{eV}$ or so. In addition, this code predicts a reasonable flux of reflected Ar neutrals
(0.25 $\times$ flux $)$ with a wide angular distribution and an average energy of $120 \mathrm{eV}$. This Ar will have a rather low sputter yield on the deposited film ( 0.1 or less). There was no evidence of edge faceting on the deposited films which would be the first observable effect of reflected-neutral sputtering of the film. It is more likely that this reflected neutral flux contributes to a general heating of the sample surface (tens of degrees $\mathrm{C}$ ) and may also lead to some level of enhanced surface diffusion, either through thermal means or by means of low-angle knock-on enhancement of the mobility of adatoms on the sample surface.

At the sample surface, the depositing metal atoms first encounter a quartz surface. The reflection dynamics of this surface can also be calculated with the TRIM code, assuming an incoming $\mathrm{Ta}$ atom at $20-30 \mathrm{eV}$. The reflection probability as a function of angle of Ta from quartz is low, as shown in Fig. 7. However, once the surface becomes covered with a metal film, the reflection dynamics change considerably. The reflection of $25 \mathrm{eV} \mathrm{Ta}$ onto a Ta surface is also shown as a function of incident angle in Fig. 7. The higher reflection probability for $\mathrm{Ta}$ on $\mathrm{Ta}$ is related to the better mass match of the projectile and the surface than in the case of Ta on quartz. In addition, it should not be surprising that sputtered, refractory atoms of such high energy are not deposited by a neargrazing impact on the surface. The surface binding energies are low, much lower than the incident kinetic energy and the momentum of these heavy particles is large. 


\section{$25 \mathrm{~cm}$ Throw} sample $A R=2.7: 1$

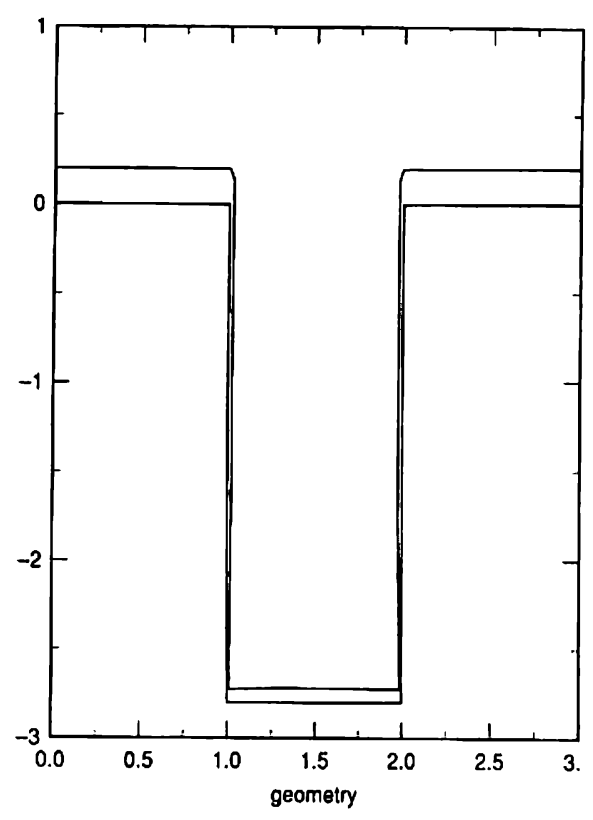

Sticking $=1.0$

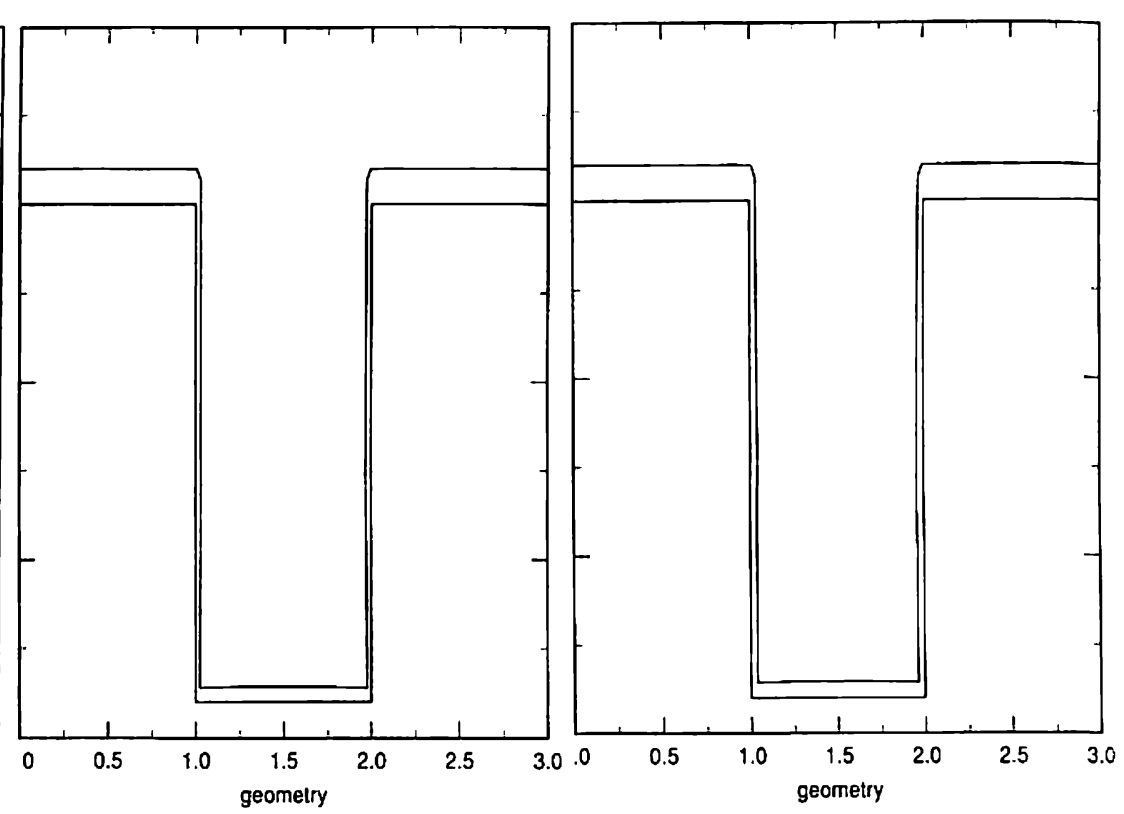

Sticking $=0.6$

FIG. 8. Predicted deposition profiles for cases consistent with Fig. $4(25 \mathrm{~cm}$ throw distances $)$ with average sticking coefficients of 1.0 .0 .8 . and 0.6 (left-to-right).

\section{B. Deposition model}

From the TRIM work, it seems likely that the primary contribution of the increased step coverage as a function of increased throw is likely to be the higher reflection probability, resulting in essentially a less-than-unity effective sticking coefficient. This conclusion was tested by using a depositionprofile computer model recently developed for I-PVD applications." For the current experiment, no ion bombardment was used. The two primary variables for these simulations were the angular distribution of the incoming flux and the effective sticking coefficient. The incoming angular distribution was designed to be a cosine distribution which has been clamped or restricted to a maximum lateral angle. This is consistent with the reduced angular arrival distribution caused by moving the sample farther and farther away from the cathode. In this case, maximum angles of $70^{\circ}, 55^{\circ}, 45^{\circ}$, $37^{\circ}$, and $31^{\circ}$ corresponded to sample distances of $5,10,15$, 20 and $25 \mathrm{~cm}$. Atoms with trajectories at higher angles than these values will not reach the sample in low pressure, long mean free path depositions and will instead deposit on the chamber sidewalls, much the same way they would be collected by a collimator in a collimated deposition.

The sticking coefficient could only be introduced in this model in an average way such that the angular dependence implied by Fig. 7 could only be approximated. In addition. the atoms which do not stick in the model are assumed to have a roughly cosine emission distribution. This is consistent with conventional adsorption and reemission from a surface. In this particular experiment, though, it is expected that there will be a significant forward-peaking to the reflected atom distribution due to the grazing-angle of incidence reflection. These two approximations will tend to underestimate the effect of reflection from the sidewalls and overestimate the reflection from the bottom surface of the trench feature. Nevertheless, the results, shown in Fig. 8, show a good qualitative correlation with the experimental observations. As the throw distance is increased, the simulations suggest that the effective sticking coefficient of the film decreased. For the experimental results shown in Fig. 5, the modeling indicates that an average, effective sticking coefficient of 0.6 is close to the experimental results. This can also be seen in Fig. 9, which plots the sidewall thicknesses of Fig. 8 in a similar format to the experimental data of Fig. 5. As the average, effective sticking coefficient is changed from 1.0 to 0.6 , the sidewall profile becomes flatter consistent with the experiment. Several artifacts are evident, though, which limit the effectiveness of this type of model. The model underestimates the net wall coverage by about $35 \%$, based possibly on the average or angle-independent sticking coeficient used. In addition, normalizing the top thickness tends to overestimate the relative changes within the trench feature. 


\section{Step Coverage Down Sidewall}

Step Coverage

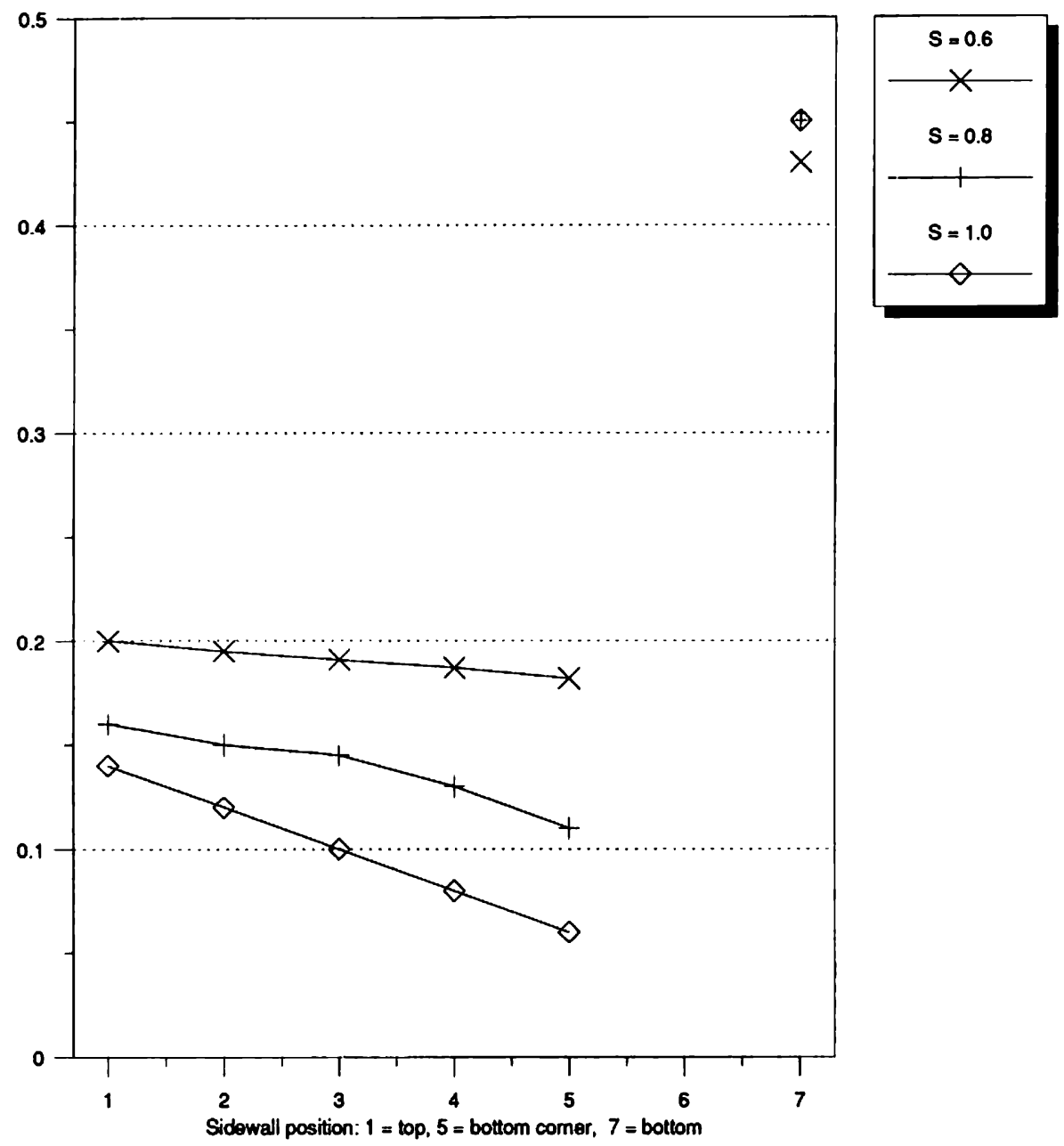

FiG. 9. Reduced data from the computer profile simulations of the sidewall and bottom step coverages as a function of throw distance and sticking coefficient.

Nevertheless. the qualitative trends predicted by the model arc quite consistent with the experimental observations.

\section{Long throw sputtering vs collimated sputtering}

In this study, the directionality of the depositing Ta atoms is set by the physical distance between the cathode and the sample. As the distance increases, the effective angular distribution narrows. This is known generically as "long throw" sputtering. This topic is routinely used with ion beam sputter deposition systems where the pressure is low $\left(10^{-4}\right.$ Torr $)$ to make individual samples. Magnetron sputtering was originally incompatible with the low pressures needed for this technique. It was first practiced using hollow-cathode enhanced magnetron discharges ${ }^{19}$ and only recently has it become practical using conventional magnetrons.

Long throw sputtering results in an intrinsic geometrical asy mmetry in the deposition thickness at the edge of a wafer. Because of the limited physical size of the cathode which is essential to any sort of long throw geometry the edge regions of the sample receive a deposition flux more from the center than from the edge and as such have a deposit which is measurably thicker on the outside sidewall of a feature than on the inside (Fig. 10).

The effect observed in this article, namely the reduction in sticking coefficient as the deposition angle becomes more grazing, might initially be expected to help alleviate the intrinsic deposition asymmetry near the wafer edge. It turns out for intermediate distances, the asymmetry is not reduced and may be enhanced. At $20 \mathrm{~cm}$ throw distance, the angular arrival distribution to the outside sidewall of a via near the edge of a wafer is $-51^{\circ}$ to $+12^{\circ}$ (from normal incidence), compared to $\pm 37^{\circ}$ in the center of the wafer. Therefore, the sticking probability on the outside sidewall (the wall which sees deposition from the center) is actually increased, resulting in thicker deposition. The inside sidewall (the wall which is deposited on only from the very edge of the cathode) has effectively a lower sticking coefficient because the deposition is more grazing. Therefore, rather than reduce the intrinsic asymmetry at the edge, the effect seen in this paper may tend to exaggerate it. Increasing the throw distance to $34 \mathrm{~cm}$ 


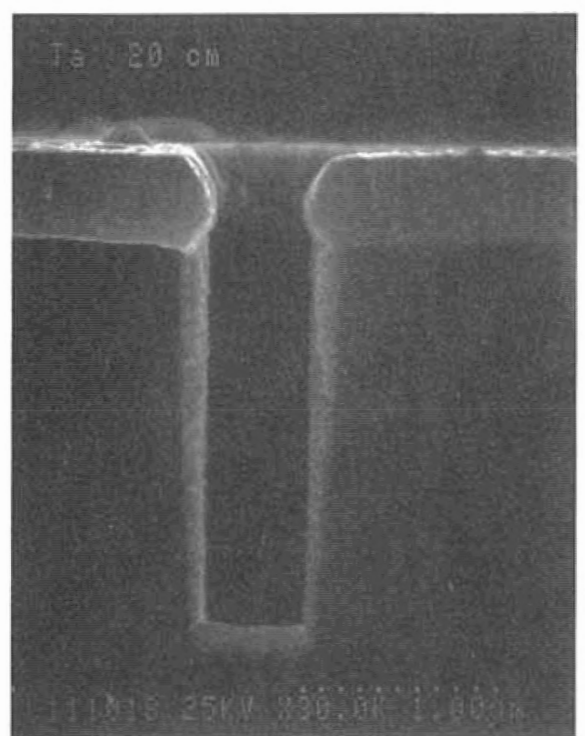

(a)

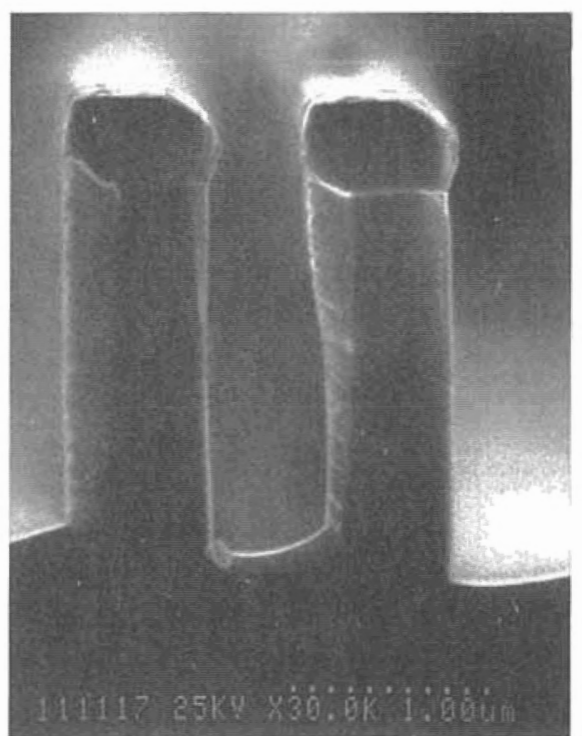

(b)

FIG. 10. (a) Via sample located at the edge of a wafer (radius $=9.5 \mathrm{~cm}$ ) for a cathode-to-sample distance of $20 \mathrm{~cm}$. The via has been cleaved in along the radial direction of the wafer. (b) A trench sample located at the wafer edge which has been cleaved in a direction tangent to the wafer edge. The centerline of the system is to the left of the trench.

changes the edge distribution to $-36^{\circ}$ to +7 . compared to $\pm 23^{\circ}$ in the center. Even with the reduced sticking of the depositing refractory metal, the cross section of vias deposited at the edge of a wafer is still clearly asymmetric (Fig. 11).

In contrast, collimated sputtering relies on the geometrical filtering of an array of holes or channels interposed between the cathode and the wafer. Each tube functions as a pinhole camera to image a specific area of the cathode onto the sample. If the erosion rate of the cathode is spatially uni-

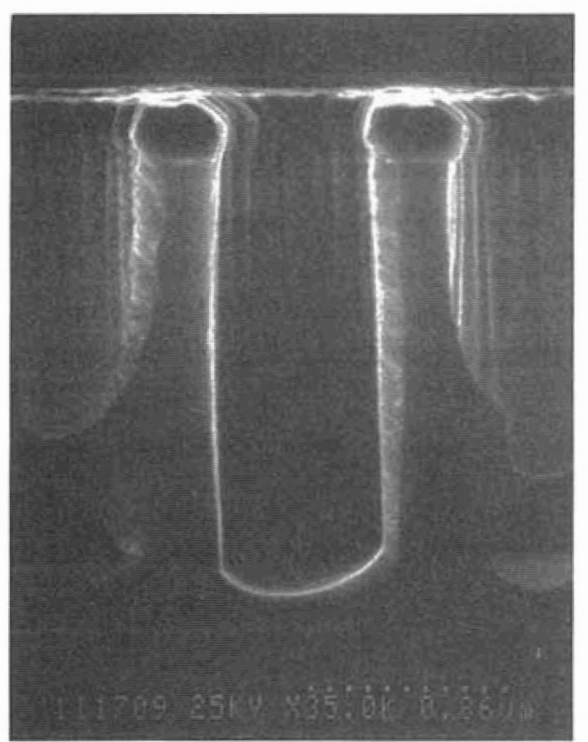

FIG. 11. Via sample located at the wafer edge for a cathode-to-sample distance of $35 \mathrm{~cm}$, cleaved in a direction tangent to the wafer edge. The centerline of the system is to the left of the via shown. form, the angular dependence of the deposit is independent of position on the wafer below the collimator.

From a practical point of view, increased directionality can easily be caused by either collimation or increased cathode-to-sample distance. The deposition rates for each technique are roughly similar, because each is a geometrical filter. The long throw system can be considered a collimator with simply one cell. Each of these techniques has its problems, though, in manufacturing applications. Collimation requires tool modification to mount the typically water-cooled collimator plates. In addition, there is generally a need to change the uniformity profile of the cathode to account for the pinhole-camera like effect of the collimator which images specific areas of the cathode onto the sample. Collimated sputtering also results in lifetime and contamination issues with the collimator and has added significant cost to the deposition of diffusion barrier or liner films. Conversely, for increases in throw distance, other changes in the cathode configuration are necessary to eliminate nonuniformities within the deposition. Because the cathodes have finite size, the angular distribution near the edge of the wafer may be different from the center of the wafer, resulting in an asymmetry to the deposition within a trench feature. This effect is partially countered by the less-than-unity effective sticking coefficient seen in this study but requires that the cathodeto-sample distance be increased much more than originally anticipated.

The implication of this work is clear and yet initially counterintuitive: in cases where the effective sticking coefficient is significantly less than one, increasing the directionality of the depositing flux will allow more redistribution of atoms during the primary deposition, which will lead in the case of a diffusion barrier application to a more conformal 
film. Therefore, increased directionality of the depositing flux. which should lead to lower sidewall step coverage and increased bottom step coverage in cases of near-unity sticking. leads instead in cases of much-less-than-unity sticking to a much more conformal deposition: with significant sidewall step coverage and a relatively small difference between the sidewall and bottom surface coverage. Without the increased directionality, this effect would not be seen because of the very strong angular dependence of the reflection coefficient.

Another implication of this work is that as the deposit becomes more directional and hence more conformal, the columnar, "zone l" microstructure typically observed with collimated sputtering on steep sidewalls can be partially suppressed. The columnar microstructure is due to the deposition of atoms occurring from a single direction. When reflection is factored in and becomes significant to perhaps $30 \%$ of the deposited flux, this single direction of deposition issue is suppressed and the films become denser and less columnar. This will result in better diffusion barrier performance. It will also reduce the thickness of the film needed for an effective diffusion barrier. Currently with $\mathrm{Ti} / \mathrm{TiN}$ technology, this thickness is on the order of $500 \AA$. This is acceptable for 0.5 - $\mu \mathrm{m}$-wide features but obviously becomes prohibitive at the feature size approaches $0.18 \mu \mathrm{m}$, late in the $256 \mathrm{Mbit}$ dynamic random access memory generation.

\section{CONCLUSIONS}

The experimental study, as well as the two computer models examined, suggest that the effective sticking probability of these refractory metal atoms, and hence the step coverage, is strongly dependent on the directionality of the incident flux. As the throw distance is increased the depositing flux becomes closer to normally incident on the sample, i.e. more directional. At the same time the flux is becoming more vertical, the probability of reflection for a particle incident on the vertical sidewall is increasing, which increases the probability that the depositing atom will rebound at least once from the steep sidewalls and land lower down into the trench feature increasing the conformality of the deposit.

'J.A. Thornton. J. Vac. Sci. Technol. 11, 666 (1974),

'D. Pramanik and V. Jain, Solid State Technol. 73-38 (1973).

'M. Wittmner, J. Vac. Sci. Technol. A 3, 1797 (1985).

${ }^{4} \mathrm{G}$. Dixit, C. Wei, and F. Liou, Appl. Phys. Lett. 62, 471 (1985).

'J.-C. Chiou and M.C. Chen, Proceedings of the VLSI Multilevel Interconnect Conference (VMIC), Santa Clara, CA, 1995 (unpublished), p. 207.

'S.M. Rossnagel, D. Mikalsen, H. Kinoshita, and J. Cuomo, J. Vac. Sci. Technol. A 9, 261 (1991).

${ }^{7} R$. Joshi and S. Brodsky. Proceedings of the VLSI Multilevel Interconnect Conference (VMIC). Santa Clara, CA, 1992 (unpublished), p. 253.

${ }^{8}$ K.C. Wang, E.J.H. Chiang. A.L. Helms, Jr., and R. Wu. in Ref. 5, p. 204.

${ }^{9}$ S.M. Rossnagel and J. Hopwood, Appl. Phys. Lett. 63, 3285 (1993).

${ }^{10}$ S.M. Rossnagel and J. Hopwood, J. Vac. Sci. Technol. B 12, 449 (1994).

I'S. Hamaguchi and S. Rossnagel. J. Vac. Sci. Technol. B 13. 183 (1995).

${ }^{12}$ P.F. Cheng, S.M. Rossnagel, and D.N. Ruzic, J. Vac. Sci. Technol. B 13. 203 (1995)

${ }^{13}$ S.M. Rossnagel, Thin Solid Films 263, 1 (1995).

${ }^{14} \mathrm{D}$. Edelstein (private communication).

${ }^{15}$ A. Leybovich, T. Kuniya, P.C. Smith. M.B. Hendricks. and D.N. Ruzic, J. Vac. Sci. Technol. A 12, 1618 (1994).

${ }^{16}$ G. K. Wehner, Phys. Rev. 114. 1270 (1959).

${ }^{17} \mathrm{D}$. Ruzic, in Handbook of Plasma Processing Techmology, edited by S.M. Rossnagel, J.J. Cuomo, and W.D. Westwood (Noyes, Park Ridge, NJ, 1990), Chap. 3.

${ }^{18}$ D.N. Ruzic, Nucl. Instrum. Methods B 47, 118 (1990).

${ }^{19}$ J.J. Cuomo and S.M. Rossnagel. J. Vac. Sci. Technol. A 4. 393 (1986). 